\title{
Analysis of the Selected Aspects of a Crash between Two Vehicles
}

\author{
Jerzy Kisilowski \\ Faculty of Transport and Electrical Engineering, \\ Kazimierz Pulaski UTH \\ Radom, Poland \\ jkisilow@kisilowscy.waw.pl
}

\author{
Jaroslaw Zalewski \\ Faculty of Administration and Social Sciences \\ Warsaw University of Technology \\ Warsaw, Poland \\ j.zalewski@ans.pw.edu.pl
}

\begin{abstract}
In the article a computer simulation of vehicle collision was prepared using the PC-Crash 8.0 software. The results of this simulation were compared to analytical calculations by Routh method with the use of the coefficient of restitution for deformations or velocities. The equations of motion during the crash were formulated in reference to the senses of the vectors. The use mesh based model of a crash was considered, for the stiffness of car bodies in both models was not known.
\end{abstract}

Analysis concerning the chosen aspects of the consequences of car crash was run, mainly due to the assumption of the roughness of vehicle bodies. The coefficient of restitution for the tangential direction was considered in order to make the crash model more realistic. (Abstract)

Keywords: road collision analysis, simulation, car crash.

\section{INTRODUCTION}

Modelling of car crash may be considered in different aspects [2], [4], [6]. In this paper attention was paid to front impact and side impact crash, as the most frequent in Poland in the period of 15 years (fig. 1). As a result of the side impact fatalities or several injuries of passengers may occur, as well as widespread damage to the car, following the change in its mass - inertia parameters.

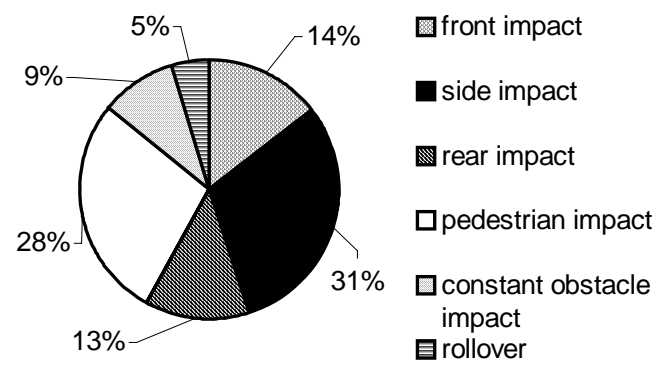

Figure 1. Participation of the selected types in general number of collisions between 1995 and 2010.

Simulations of the oblique side impact and the frontal oblique impact were conducted using the PCCrash 8.0. The vehicle model being struck is Volkswagen Passat 2.0, while the striking one - Opel Vectra 2.2 DTI, both from 2002 - 2005 years of production. Simulation of a collision was prepared at high velocity on the equal the intersection, and on a straight section of single road. This case reflects the event in which Volkswagen was struck at the intersection of enforcing the right of way. In the event of a frontal collision Opel left its lane for the one of the opposite direction.

\section{ASSUMPTIONS}

Duration time of the simulation was $2 \mathrm{~s}$, of which the longevity of the impact itself was 0,9s. Basic assumptions for both simulations were as given:

- vehicle models are linear, the vehicle bodies are treated as rectangular element or sets of rectangular elements having constant stiffness;

- motion takes place on a dry surface with a coefficient of adhesion equal to 0.8 ;

- the vehicles are moving at velocities: Passat at $50 \mathrm{~km} / \mathrm{h}$, Vectra at $80 \mathrm{~km} / \mathrm{h}$;

- the initial mass of the Volswagen Passat amounting $1370 \mathrm{~kg}$ has been increased by the weight of the driver and passengers equal to $272 \mathrm{~kg}$ in accordance with [1], while the initial mass of the Opel Vectra amounting $1510 \mathrm{~kg}$ has been increased by the weight of the driver and three passengers equal to $272 \mathrm{~kg}$, also in accordance with [1]. No baggage was included;

- according to [1] the height of the center of mass for the laden vehicle was assumed at $0.56 \mathrm{~m}$ for both Opel and Volkswagen;

Assuming that the driver and passenger both weigh at $68 \mathrm{~kg}$ is consistent with the procedures for determining the allowed load of a car described in [1].

In fig. 2 location of both cars before and after the side impact collision is shown, whereas in fig. 3 - before and after the front impact collision.

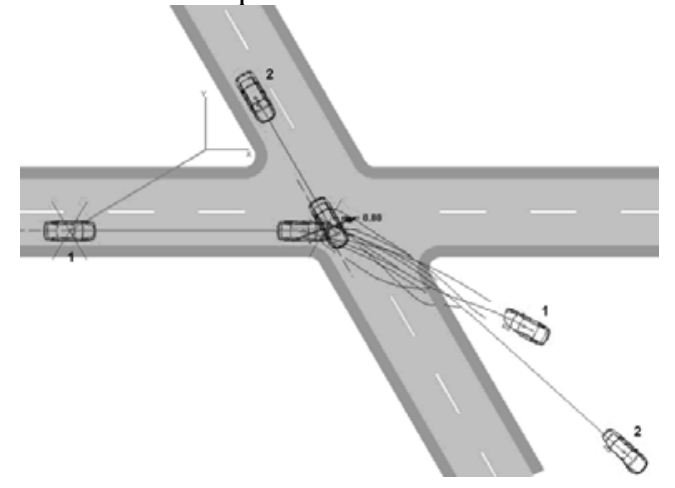

Figure 2. Location of vehicles before and after side impact, where 1-Opel, 2 - Volkswagen. 


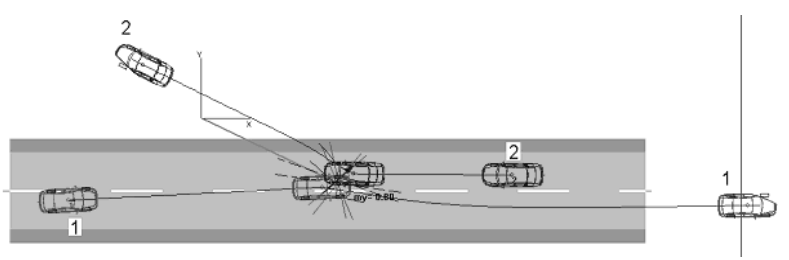

Figure 3. Location of vehicles before and after front impact, where 1 - Opel, 2 - Volkswagen.

The main aim of the computer simulation was to examine the motor vehicles collision with the defined parameters as well as verification of the simulation results through analytical calculations. Another aim was to check the differencies in simulation results after running it for several times. So called "use mesh based impact model" was used. It is a model of impact, where a vehicle body is divided into little polygons and the kinetic energy of impact is into the deformation of each polygon.

For the problem of contact of the vehicle rough surfaces the coefficient of restitution was analysed, for which the prepared calculations would result the same as the simulation.

\section{DESCRIPTION OF THE SIMULATION AND THE SELECTED ELEMENTS OF PC-CRASH SOFTWARE}

In PC-Crash a vehicle is depicted as a single rigid block [3], where the body is represented by a rectangular prism of a certain mass, moments of inertia, structural* and torsional stiffness.

The parameter defined as the stiffness of the body in PC-Crash is described as a depth of deformations in relation to the weight of the vehicle. Wheel stiffness is half the value of the vehicle body, whereas the roof plate in models of passenger cars is assumed 75 percent less stiff than the stiffness of the lower vehicle body.

In table 4 selected parameters of the protocol for the initial and final phase of the collision are shown with the segmentation for the side and front impact. Noticeable is the depth of the body deformation, which in the side impact velocity of about $80 \mathrm{~km} / \mathrm{h}$ is $0.34 \mathrm{~m}$ for Volkswagen, bearing in mind the so-called mutual penetration of the vehicle bodies. Front of the car striking (Opel) was strongly deformed and the deformation depth is $0.40 \mathrm{~m}$ This shows that for such type of collision and for cars of similar weight and size the depth of deformation is larger for the front of the impacting vehicle (rigid passenger compartment). Confirmation of this can be found in section on the front impact collision. For a vehicle moving at higher velocities (Vectra) the depth of deformation is greater. It

\footnotetext{
* structural stiffness according to [3] is defined on the basis of the depth of deformation associated to the weight of the car at a resting position.
}

follows that the impact velocity affects the resulting strain, which is consistent with the basic equations of the theory of collisions [6], [7].

TABLE I. THE PROTOCOL OF SIDE AND FRONT IMPACT CRASH. IN BUCKETS THE MOMENTS OF INERTIA AFTER THE LOAD WITH PASSENGERS ARE SHOWN.

\begin{tabular}{|c|c|c|c|c|}
\hline & \multicolumn{2}{|c|}{$\begin{array}{c}\text { Side impact } \\
\text { crash }\end{array}$} & \multicolumn{2}{|c|}{$\begin{array}{c}\text { Front impact } \\
\text { crash }\end{array}$} \\
\hline car & $\begin{array}{c}\text { Opel } \\
\text { Vectra }\end{array}$ & $\begin{array}{c}\text { VW } \\
\text { Passat }\end{array}$ & $\begin{array}{c}\text { Opel } \\
\text { Vectra }\end{array}$ & $\begin{array}{c}\text { VW } \\
\text { Passat }\end{array}$ \\
\hline \multicolumn{5}{|c|}{ VALUES AT THE BEGINNING OF THE CRASH } \\
\hline $\begin{array}{c}\text { before crash velocity } \\
{[\mathrm{km} / \mathrm{h}]}\end{array}$ & 76.49 & 48.22 & 75.23 & 45.13 \\
\hline vehicle angle [deg.] & -1.27 & -60.40 & 2.79 & 178.61 \\
\hline $\begin{array}{l}\text { velocity direction (ni) } \\
\text { [deg.] }\end{array}$ & -2.76 & 278.76 & 0.61 & 174.88 \\
\hline $\begin{array}{c}\text { angular velocity } \\
\text { around } \mathrm{z} \text { axis (om) } \\
{[1 / \mathrm{s}]}\end{array}$ & -1.11 & -0.24 & -0.25 & -0.90 \\
\hline $\begin{array}{c}\text { moment of inertia } \\
\text { around } \mathrm{x} \text { axis }\left[\mathrm{kgm}^{\wedge} 2\right]\end{array}$ & \multicolumn{2}{|c|}{$714(822.98)$} & \multicolumn{2}{|c|}{$661.9(771.54)$} \\
\hline $\begin{array}{c}\text { moment of inertia } \\
\text { around y axis }\left[\mathrm{kgm}^{\wedge} 2\right]\end{array}$ & \multicolumn{2}{|c|}{$2379.9(2743.16)$} & \multicolumn{2}{|c|}{$\begin{array}{c}2206.2 \\
(2571.66)\end{array}$} \\
\hline $\begin{array}{c}\text { moment of inertia } \\
\text { around } \mathrm{z} \text { axis }\left[\mathrm{kgm}^{\wedge} 2\right]\end{array}$ & \multicolumn{2}{|c|}{$2379.9(2743.16)$} & \multicolumn{2}{|c|}{$\begin{array}{c}2206.2 \\
(2571.66)\end{array}$} \\
\hline $\begin{array}{l}\text { impulse of the impact } \\
\text { force [Ns] }\end{array}$ & \multicolumn{2}{|c|}{13096.46} & \multicolumn{2}{|c|}{7396.46} \\
\hline \multicolumn{5}{|c|}{ VALUES AT THE END OF THE CRASH } \\
\hline $\begin{array}{c}\text { after crash velocity } \\
{[\mathrm{km} / \mathrm{h}]}\end{array}$ & 52.93 & 63.07 & 64.39 & 35.59 \\
\hline vehicle angle [deg.] & -1.27 & -60.40 & 2.79 & 178.61 \\
\hline $\begin{array}{l}\text { velocity direction (ni) } \\
\text { [deg.] }\end{array}$ & -13.97 & 332.15 & -7.87 & 156.04 \\
\hline $\begin{array}{c}\text { angular velocity } \\
\text { around } \mathrm{z} \text { axis (om) } \\
{[1 / \mathrm{s}]}\end{array}$ & -3.04 & 1.31 & -1.17 & -2.16 \\
\hline $\begin{array}{c}\text { depth of deformations } \\
{[\mathrm{m}]}\end{array}$ & 0.40 & 0.34 & 0.63 & 0.55 \\
\hline $\begin{array}{l}\text { coefficient of } \\
\text { restitution } \mathrm{k}\end{array}$ & \multicolumn{2}{|c|}{0.10} & \multicolumn{2}{|c|}{0.10} \\
\hline
\end{tabular}

The values of the angular velocities around the vertical axes in the initial phase of collision were different from zero. Following the adoption of the center of mass of vehicles greater than zero, there occur phenomena of roll, and the moments of inertia for all axes are included [3]. 


\section{VERIFICATIONOF THE OBTAINED RESULTS ON THE BASIS OF ANALYTICAL CALCULATIONS}

For comparison to the simulation results calculations were prepared according to the method for the collision issues including the tangential velocity restitution. Data for the calculation was assumed according to the table 4, however the input of tangential and normal velocities were obtained by the transition from the cartesian coordinate system (fig. 4) to the natural local coordinate system (tangential and normal, fig. 5, 6). Velocity vector of the striking vehicle (Opel) had to be projected orthogonally on the axes adopted in accordance with fig. 6a. It was assumed that the velocity vector of the impacted vehicle is parallel to the tangent axis ( $\mathrm{t}$ ), and the striking vehicle vector coincides with the normal to the collision (n).

In the side impact, the angle of the vehicle taken from the table 4 was assumed as the impact angle, since it is measured relative to the $\mathrm{x}$ axis in the Cartesian coordinate system $x-y$. It is also easy to situate the tangential axis (along the edge) of the struck vehicle and the normal axis (perpendicular to the tangent). It is more difficult to realize this for a frontal collision, where the vehicles are positioned almost parallel to the $\mathrm{x}$ axis. Using trigonometry, knowing the distance of the vehicles from the center of collision (fig. 6b) the angles to the axes $\mathrm{x}$ and $\mathrm{y}$ can be calculated.
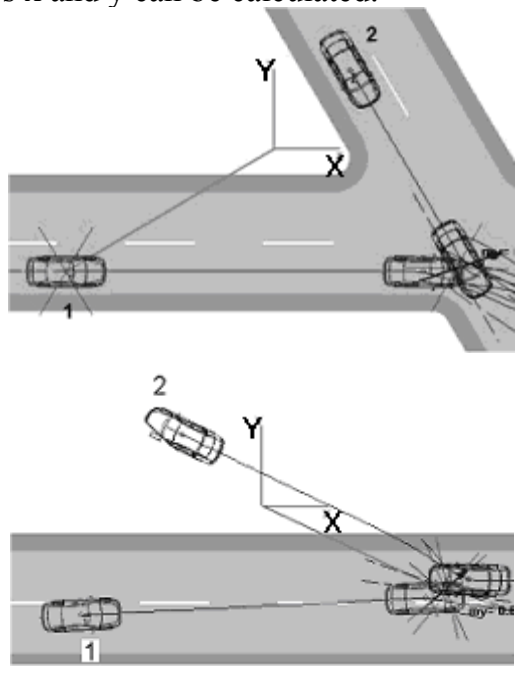

Figure 4. Coordinate system x-y for side and front impact.

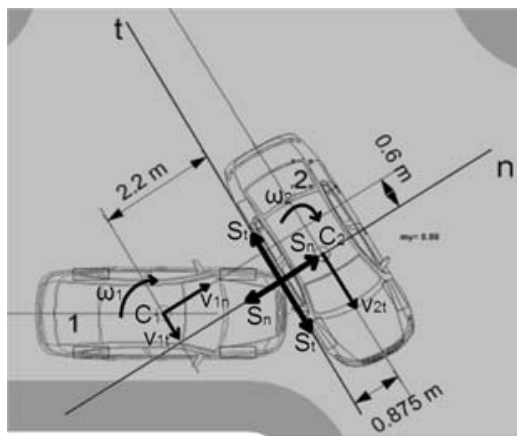

Figure 5. Location of the local coordinate system for the side impact with the velocities.

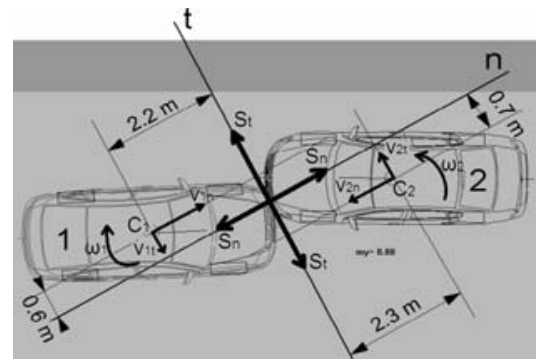

Figure 6. Location of the local coordinate system for the front impact with the velocities.

Based on crash theory between bodies [4] concerning collision theory, theoretical analysis on the issue of vehicle collision was adapted. The issue of restitution of tangential velocity was taken into account. It was assumed that during the collision except for the so-called volumetric strains, there occur also non-dilatational strains associated with the stress in the tangential direction occurring on the surfaces of vehicles. In analogy to Newton's hypothesis the formula to determine the coefficient of restitution of tangential velocities [2] is presented.

$$
\theta=\frac{w_{t}^{\prime}}{w_{t}}
$$

The relative tangential velocity in the nonslip collision was described by the formula (4.2).

$$
w_{t}^{\prime} \equiv w_{t}-\alpha_{t t} S_{t}-\alpha_{n t} S_{n}=\theta w_{t}
$$

The relative normal velocity

$$
w_{n}-\alpha_{n t} S_{t}-\alpha_{n n} S_{n}=-R w_{n}
$$

where [4]:

$$
\begin{aligned}
& \alpha_{t t}=\frac{1}{m_{1}}+\frac{1}{m_{2}}+\frac{n_{1}^{2}}{I_{1}}+\frac{n_{2}^{2}}{I_{2}} \\
& \alpha_{n n}=\frac{1}{m_{1}}+\frac{1}{m_{2}}+\frac{t_{1}^{2}}{I_{1}}+\frac{t_{2}^{2}}{I_{2}} \\
& \alpha_{n t}=\alpha_{t n} \equiv \frac{n_{1} t_{1}}{I_{1}}-\frac{n_{2} t_{2}}{I_{2}}
\end{aligned}
$$

$m_{1}, m_{2}$ - the mass of vehicle 1 and 2 respectively;

$n_{1}, n_{2}, t_{1}, t_{2}$ - the normal and tangenial coordinates of the center of mass for both vehicles in relation to the center of crash (the initial point of contact); 
$I_{1}, I_{2}$ - the moments of inertia around the vertical axes passing throug the center of mass of every vehicle.

Equations (4.2) and (4.3) were solved with respect to impulses, which in turn allowed determining the value of the tangential and normal impulses (4.5).

$$
\begin{aligned}
& S_{t}=\frac{-(1+R) \alpha_{n t} w_{n}+(1-\theta) \alpha_{n n} w_{t}}{\alpha_{n n} \alpha_{t t}-\alpha_{n t}^{2}} \\
& S_{n}=\frac{(1+R) \alpha_{t t} w_{n}-(1-\theta) \alpha_{n t} w_{t}}{\alpha_{n n} \alpha_{t t}-\alpha_{n t}^{2}}
\end{aligned}
$$

Solution of this problem for a car crash on rough surfaces requires knowledge of three factors: the dynamic coefficient of friction $f$ (in the Routh system for the transient impulse $\mathrm{St}=f \mathrm{Sn}$, if of course the crash occurs with a slip between the surfaces of vehicles taking part), the coefficient of restitution for normal $\mathrm{R}$ and tangential velocities.

In general the equations of motion during the crash can be presented as:

$$
\begin{aligned}
& m_{i}\left(v_{i n}^{\prime}-v_{i n}\right)= \pm S_{n} \\
& m_{i}\left(v_{i t}^{\prime}-v_{i t}\right)= \pm S_{t} \\
& I_{i}\left(\omega_{i}^{\prime}-\omega_{i}\right)= \pm S_{t} n_{i} \pm S_{n} t_{i}
\end{aligned}
$$

where:

${ } m_{i}$ - mass of the vehicle no i;

- $v_{i t}$ - tangential velocity of the vehicle no $\mathrm{i}$;

- $v_{\text {in }}$ - normal velocity of the vehicle no $\mathrm{i}$;

- $\omega_{i}$ - angular velocity of the vehicle no $\mathrm{i}$;

- $I_{i}$ - moment of inertia of the vehicle no i around the vertical;

- $S_{n}, S_{t}$ - normal and tangential impulses of the crash force;

For the two presented cases kinematical state after the collision is described by formulas, respectfully for the side and front impact crash:

$$
\begin{array}{ll}
v_{1 t}^{\prime}=v_{1 t}+\frac{S_{t}}{m_{1}}, & v_{1 t}^{\prime}=v_{1 t}-\frac{S_{t}}{m_{1}}, \\
v_{1 n}^{\prime}=v_{1 n}-\frac{S_{n}}{m_{1}}, & v_{1 n}^{\prime}=v_{1 n}-\frac{S_{n}}{m_{1}}, \\
\omega_{1}^{\prime}=\omega_{1}+\frac{S_{t} n_{1}}{I_{1}}+\frac{S_{n} t_{1}}{I_{1}}, & \omega_{1}^{\prime}=\omega_{1}+\frac{S_{t} n_{1}}{I_{1}}-\frac{S_{n} t_{1}}{I_{1}}, \\
v_{2 t}^{\prime}=v_{2 t}-\frac{S_{t}}{m_{2}}, & v_{2 t}^{\prime}=v_{2 t}-\frac{S_{t}}{m_{2}}, \\
v_{2 n}^{\prime}=v_{2 n}-\frac{S_{n}}{m_{2}}, & v_{2 n}^{\prime}=v_{2 n}-\frac{S_{n}}{m_{2}}, \\
\omega_{2}^{\prime}=\omega_{2}+\frac{S_{t} n_{2}}{I_{2}}+\frac{S_{n} t_{2}}{I_{2}} . \omega_{2}^{\prime}=\omega_{2}-\frac{S_{t} n_{2}}{I_{2}}-\frac{S_{n} t_{2}}{I_{2}} .
\end{array}
$$

Distances $\mathrm{n}_{1}, \mathrm{n}_{2}, \mathrm{t}_{1}, \mathrm{t}_{2}$ of both centers of mass to the center of collision were measured in PC-Crash with both cars located at the point of initial contact position. In shear and compression instantaneous velocity formulas [4], the instantaneous values were replaced by the values from the beginning of the collision. Then the coefficients $\mathrm{f}, \mathrm{R}$, and $\theta$ were so chosen, that the values of after crash velocities were as close as possible to those obtained in the simulation.

The calculations were prepared with the use of MS Excel. They were conducted in two directions.

\section{A. Firtst approach}

First it seemed necessary to obtain the exact values of the crash impulses as in the protocol (table 4) in order to examine to what extent the values of the after crash velocities and the coefficients would be changed, without respect to the values of the three analyzed coefficients.

For the side impact crash the results are as follows. Respectfully before and after the crash:

$$
\begin{array}{ll}
v_{1 n}=10.62 \frac{\mathrm{m}}{\mathrm{s}} ; v_{1 t}=18.4 \frac{\mathrm{m}}{\mathrm{s}} ; & v_{1 n}^{\prime}=17.12 \frac{\mathrm{m}}{\mathrm{s}} ; v_{1 t}^{\prime}=21.83 \frac{\mathrm{m}}{\mathrm{s}} \\
v_{2 n}=0 ; v_{2 t}=13.39 \frac{\mathrm{m}}{\mathrm{s}} ; & v_{2 n}^{\prime}=7.05 \frac{\mathrm{m}}{\mathrm{s}} ; v_{2 t}^{\prime}=9.66 \frac{\mathrm{m}}{\mathrm{s}} \\
\omega_{1}=-1.11 \frac{1}{\mathrm{~s}} ; \omega_{2}=-0.24 \frac{1}{\mathrm{~s}} ; & \omega_{1}^{\prime}=1.26 \frac{1}{\mathrm{~s}} ; \omega_{2}^{\prime}=1.84 \frac{1}{\mathrm{~s}}
\end{array}
$$

The whole impulse $\mathrm{S}=13096$ Ns.

With coefficients $f=0.52, R=0.21$ and $\theta=-0.85$ the values of velocities in the final phase of collision were:

$$
v_{1}^{\prime}=99.89 \frac{\mathrm{km}}{\mathrm{h}}, v_{2}^{\prime}=43.07 \frac{\mathrm{km}}{\mathrm{h}},
$$

compared to:

$$
v_{1}^{\prime}=52.93 \frac{\mathrm{km}}{\mathrm{h}}, v_{2}^{\prime}=63.07 \frac{\mathrm{km}}{\mathrm{h}}
$$

obtained as a result of

the simulation.

For the front impact crash the results are as follows. Respectfully before and after the crash: 


$$
\begin{aligned}
& v_{1 t}=12.57 \frac{m}{s} ; v_{1 n}=16.68 \frac{m}{s} ; v_{1 t}^{\prime}=8.85 \frac{m}{s} ; v_{1 n}^{\prime}=14.84 \frac{m}{s} \\
& v_{2 n}=10.51 \frac{m}{s} ; v_{2 t}=6.82 \frac{m}{s} ; v_{2 n}^{\prime}=8.51 \frac{m}{s} ; v_{2 t}^{\prime}=2.79 \frac{m}{s} \\
& \omega_{1}=-0.25 \frac{1}{s} ; \omega_{2}=-0.9 \frac{1}{s} ; \quad \omega_{1}^{\prime}=4.34 \frac{1}{s} ; \omega_{2}^{\prime}=-7.72 \frac{1}{s}
\end{aligned}
$$

The whole impulse $\mathrm{S}=7396 \mathrm{Ns}$.

With coefficients $f=0.71, R=-1.59$ and $\theta=-2.89$ the values of velocities in the final phase of collision were:

$$
v_{1}^{\prime}=62.23 \frac{\mathrm{km}}{\mathrm{h}}, v_{2}^{\prime}=32.26 \frac{\mathrm{km}}{\mathrm{h}} \text {, }
$$

compared to:

$$
v_{1}^{\prime}=64.39 \frac{\mathrm{km}}{\mathrm{h}}, v_{2}^{\prime}=35.59 \frac{\mathrm{km}}{\mathrm{h}}
$$

obtained as a result of the simulation.

From the obtained results it can be concluded that when aiming to obtain the values of impulses equal to those from the simulation of side impact crash it is not possible to obtain the same values of after crash velocities. However, in the case of front crash the results are close.

When examining the analyzed coefficients it is easy to notice, that for the side crash their values may be possible. The question, however, is what values can the $\mathrm{R}$ coefficient have in the side impact.

In the front impact the values of both $\mathrm{R}$ and $\theta$ coefficients seem to be unlikely and it may indicate, that their values in the real conditions may be close to zero for $R$ and -1 for $\theta$ (see chapter 5).

\section{B. Second approach}

As the next step it seemed essential to obtain the final values of after crash velocities close or equal to those in crash protocol (table 4) without respect to the values of the three analyzed coefficients.

For the side impact crash the results are as follows. Respectfully before and after the crash:

$$
\begin{aligned}
& v_{1 n}=10.62 \frac{m}{s} ; v_{1 t}=18.4 \frac{m}{s} ; v_{1 n}^{\prime}=5.35 \frac{m}{s} ; v_{1 t}^{\prime}=15.5 \frac{m}{s} \\
& v_{2 n}=0 ; v_{2 t}=13.39 \frac{m}{s} ; \quad v_{2 n}^{\prime}=-5.71 \frac{m}{s} ; v_{2 t}^{\prime}=16.54 \frac{m}{s} ; \\
& \omega_{1}=-1.11 \frac{1}{s} ; \omega_{2}=-0.24 \frac{1}{s} ; \omega_{1}^{\prime}=-3.19 \frac{1}{s} ; \omega_{2}^{\prime}=-1.99 \frac{1}{s}
\end{aligned}
$$

The whole impulse $\mathrm{S}=10713 \mathrm{Ns}$.

With coefficients $f=0.55, R=-1.97$ and $\theta=2.59$ the values of velocities in the final phase of collision were:

$$
\begin{aligned}
& v_{1}^{\prime}=59.04 \frac{\mathrm{km}}{\mathrm{h}}, v_{2}^{\prime}=63 \frac{\mathrm{km}}{\mathrm{h}}, \\
& \text { compared to: } \\
& v_{1}^{\prime}=52.93 \frac{\mathrm{km}}{\mathrm{h}}, v_{2}^{\prime}=63.07 \frac{\mathrm{km}}{\mathrm{h}}
\end{aligned}
$$

obtained as a result of
For the front impact crash the results are as follows. Respectfully before and after the crash:

$$
\begin{aligned}
& v_{1 t}=12.57 \frac{\mathrm{m}}{\mathrm{s}} ; v_{1 n}=16.68 \frac{\mathrm{m}}{\mathrm{s}} ; v_{1 t}^{\prime}=10.86 \frac{\mathrm{m}}{\mathrm{s}} ; v_{1 n}^{\prime}=14.41 \frac{\mathrm{m}}{\mathrm{s}} ; \\
& v_{2 n}=10.51 \frac{\mathrm{m}}{\mathrm{s}} ; v_{2 t}=6.82 \frac{\mathrm{m}}{\mathrm{s}} ; v_{2 n}^{\prime}=8.05 \frac{\mathrm{m}}{\mathrm{s}} ; v_{2 t}^{\prime}=4.97 \frac{\mathrm{m}}{\mathrm{s}} \\
& \omega_{1}=-0.25 \frac{1}{\mathrm{~s}} ; \omega_{2}=-0.9 \frac{1}{\mathrm{~s}} ; \quad \omega_{1}^{\prime}=1.3 \frac{1}{\mathrm{~s}} ; \omega_{2}^{\prime}=-4.72 \frac{1}{\mathrm{~s}}
\end{aligned}
$$

The whole impulse $\mathrm{S}=5063$ Ns.

With coefficients $f=0.71, R=-0.74$ and $\theta=-1.84$ the values of velocities In the final phase of collision were:

$$
v_{1}^{\prime}=65 \frac{\mathrm{km}}{\mathrm{h}}, v_{2}^{\prime}=34.06 \frac{\mathrm{km}}{\mathrm{h}},
$$

compared to:

$$
v_{1}^{\prime}=64.39 \frac{\mathrm{km}}{\mathrm{h}}, v_{2}^{\prime}=35.59 \frac{\mathrm{km}}{\mathrm{h}}
$$

obtained as a result of the simulation.

As it can be seen from the calculations for the second approach, the coefficient of restitution of the normal velocities in both cases is well below zero, which may indicate that in real conditions its value oscillates around this value. The default value of $\mathrm{R}$ assumed in PC-Crash is 0.1 .

Moreover, the values of the angular velocities of both vehicles are much larger than those given in the protocol of computer simulation. Progressive values of impact velocities in the final phase differ slightly from the results of simulation in PC-Crash, however the total values of both impulses is smaller by about $3000 \mathrm{Ns}$ for the side impact and $2300 \mathrm{Ns}$ for the front impact to the value obtained in the simulation.

\section{V.SELECTED ASPECTS OF THE COEFFICIENT OF TANGENIAL VELOCITIES}

For better understanding of the complicated vehicle crash process it seems neccessary to be familiar with the phenomena of friction between the surfaces of the colliding vehicles. Therefore, apart from the classic coefficient of restitution $R$, it is essential to implement the coefficient for tangential velocities $\theta$, which corresponds with the so-called shape deformations of the motor vehicle body, contrary to volume deformations concerned when defining $R$.

The theory of the coefficient $\theta$ is presented in [4] and concerns occurring circular contact area, where normal stress has a spherical shape. The spread of the shear stress steming from the tangential impulse is also spherical, provided that friction between surfaces is fully developed.

Results of research on this subject shown, that when colliding steel spheres, the value of $\theta$ depends both on $\mathrm{R}$ and the angle of collision $\alpha$. On the basis of these conlcusions the formula used for calculating the value of $\theta$ can be presented as:

the simulation. 


$$
\theta=1-\frac{7}{2}(1+R) \frac{\tan \alpha_{1}^{\prime}}{\tan \alpha_{2}^{\prime}}
$$

where:

$\alpha_{1}^{\prime}$ - the angle of the vehicle no 1 after collision;

$\alpha_{2}^{\prime}$ - the angle of the vehicle no 2 after collision.

In [8] and [9] both normal and tangential coefficients of restitution were discussed. As a result of crash tests of 32 vehicles the values of normal and tangential coefficients were computed. For the vehicle masses between 566 to $1268 \mathrm{~kg}$ and velocities from 16,3 to $62,3 \mathrm{~km} / \mathrm{h}$ these values were as follows:

- normal restitution coefficient $\mathrm{R}=(-0,32 \div 0,51)$;

- tangential restitution coefficient $\theta=(-0,87 \div 0,27)$.

The results based on the tests of real vehicles seem to be in disagree with the theory, according to which the value of the normal coefficients of restitution is between 0 and 1.

\section{CONCLUSIONS}

The obtained results can be used for further research on the complicity of the vehicle collision process. One aspect of may be examination of the influence of selected crash parameters on the process of collision, whereas the other - the influence of mass - inertia disturbances in car body on stability and dynamics of so damaged and then improperly repaired vehicle [5].

Both the coefficient of restitution of normal and tangential velocity plays an important role in modeling the collision of vehicles in real conditions. The values of both coefficients depend on the type of collision and the angle of the impact force. The value of the impact force impulse was considered particularly in the case of side impact, because for such type of collision it is difficult to determine the point of impact force application.

Such qualitative evaluation may lead to arbitrary assumptions adopted in the quantitative sense, but with reference to reality.

\section{REFERENCES}

[1] Reimpell J., Podwozia samochodów, WKŁ, Warszawa 2004. (in Polish)

[2] Wicher J., Stawicki R., Modelowanie zderzenia samochodów, Zeszyty Naukowe Instytutu Pojazdów, PW, SIMR, 1(31)/99. (in Polish)

[3] PC-Crash a simulation program for vehicle accidents, Operating Manual, Version 8.0, Dr Steffan Datentechnik, Linz, Austria 2006.

[4] Gryboś R., Teoria uderzenia w dyskretnych układach mechanicznych, PWN, Warszawa 1969. (in Polish)

[5] Zalewski J. Modelowanie wpływu zaburzeń geometrii nadwozia na stateczność ruchu pojazdu samochodowego, Doctoral Thesis, Warszawa, 2011. (in Polish)

[6] Huang M., Vehicle crash mechanics, Hardbound, 2002.

[7] Osiński Z., Mechanika ogólna, PWN, Warszawa 2000. (in Polish)

[8] Wicher J., O współczynniku restytucji w zderzeniach samochodów, Zeszyty Naukowe Politechniki Świętokrzyskiej, Mechanika 84, Kielce 2006. (in Polish)

[9] Ishikawa H., Impact model for accident reconstruction - normal and tangential restitution coefficients, SAE paper 930654. 